\title{
Effect of CXCR4 and CD133 Co-expression on the Prognosis of Patients with Stage II III Colon Cancer
}

\author{
Xiao-Feng Li ${ }^{1 \&}$, Xiao-Guang Guo ${ }^{2 \& *}$, Yong-Yan Yang ${ }^{1}$, Ai-Yong $\mathrm{Liu}^{3}$
}

\begin{abstract}
Background: To explore the relationship between CXCR4, CD133 co-expression and clinicopathological features as well as prognosis of patients with phase II III colon cancer. Materials and Methods: Forty-nine paraffin-embedded samples of tumor tissue and epithelial tissue adjacent to cancer were collected from patients with colon cancer undergoing radical surgery in Baotou Cancer Hospital from January, 2010 to June, 2011. CXCR4 and CD133 expression was detected using immunohistochemistry and its relationship with clinicopathological features and the 3-year survival rate was analyzed. Results: In the tumor tissue and colonic epithelial tissue adjacent to cancer, the positive expression rates of CXCR4 were respectively $61.2 \%(30 / 49)$ and 8.16\% (4/49), while those of CD133 being 36.7\% (18/49) and 6.12\% (3/49). CXCR4 and CD133 expression in tumor tissue was not related to patient age, gender, primary focal sites, tumor size, TNM staging, histological type, tumor infiltration depth and presence or absence of lymphatic metastasis, but CXCR4 and CD133 co-expression was associated with TNM staging and lymphatic metastasis. The 3-year survival rate of patients with CXCR4 and CD133 co-expression was $27.3 \%(3 / 11)$, and that of the remainderwas $76.3 \%(29 / 38)$, the difference being significant $\left(\chi^{2}=7.0206, p=0.0081\right)$. Conclusions: $\mathrm{CXCR} 4$ and $\mathrm{CD133}$ co-expression may be a risk factor for poor prognosis of patients with stage II III colon cancer.
\end{abstract}

Keywords: CXCR4 - CD133 - co-expression - colon cancer - prognosis

Asian Pac J Cancer Prev, 16 (3), 1073-1076

\section{Introduction}

Colon cancer, one of the most common malignant gastrointestinal tumors, can be accompanied by lymphatic metastasis at the early stage, and liver and lung metastases as well as abdominal dissemination frequently occur at its advanced stage. The major causes for the death of patients with colon cancer include local recurrence, lymphatic and hematogenous disseminated metastases (Wu et al., 2014; Wu et al., 2013; Yang et al., 2013; Gong et al., 2014). The report revealed that cytokine receptors not only affected lymphatic and hematogenous disseminated metastases of tumor cells directly, but also influenced metastatic sites and processes of different tumors (Nakata et al., 2008). Tumor cells realized organ-specific metastasis based on chemokine receptors, namely some sort of chemokine receptors were highly expressed in tumor cells, whereas their corresponding chemokines were highly expressed in target organs which realized a directed metastasis from tumor cells to itself by virtue of specific binding capability of chemokine receptors and chemokines (Gupta et al., 2014).

Chemokine receptor CXCR4 is an important factor of tumor cell adhesion and migration, and its natural ligand is stromal-derived factor-1 (SDF-1), also termed CXCL12. At present, it is considered that CXCL12-
CXCR4 biological axis is a key mechanism of mediating tumor invasion and metastasis. After specific binding of CXCL12 to its receptor CXCR4 in tumor cells, various signal pathways of tumor cells can be activated, which plays an important role in tumor invasion, progression and metastasis (Li et al., 2014). CD133, one of the tumor stem cell markers, is obviously related to tumor clinical pathology and prognosis (Bahnassy et al., 2014; Liu et al., 2014). In this study, CXCR4 and CD133 expression was analyzed in colon cancer, and the relationship between their co-expression and metastasis, prognosis of patients with phase II $\sim$ III colon cancer was investigated.

\section{Materials and Methods}

\section{General data}

Forty-nine paraffin-embedded samples of tumor tissue and epithelial tissue adjacent to cancer were collected from the patients with colon cancer undergoing radical operation of colon cancer in Baotou Cancer Hospital from Jan, 2010 to Jun, 2011. All the patients with colon cancer diagnosed by histopathology and/or cytology were not given neoadjuvant chemotherapy or radiotherapy before operation, in which males and females were 28 and 21 cases, respectively. They were at the age of $31 \sim 69$, with the mean age of $(48.94 \pm 11.57)$. According 
to histological types, there were 5 cases with high differentiation (tubular adenocarcinomaIand polypoid adenocarcinoma), 24 with moderate differentiation (tubular adenocarcinomaIIand mucinous adenocarcinoma) and 20 with poor differentiation (tubular adenocarcinoma III and undifferentiated carcinoma). In terms of TNM staging, there were 24 and 25 cases respectively at phase II and III. The serosa and tissue outside serosa in 47 cases were invaded, and the invasion in 2 cases was not beyond the muscular layer. Besides, there were 32 and 17 cases respectively with and without lymphatic metastasis.

\section{Methods}

CXCR4 and CD133 expression in both colon cancer tissue and epithelial tissue adjacent to cancer was detected using immunohistochemical method. Specific operations were as follows: firstly, take paraffin-embedded samples and cut them into $4 \mu \mathrm{m}$ slices; secondly, routinely apply xylene for dewaxing, gradient alcohol for hydration and $3 \mathrm{~mol} / \mathrm{L}$ of hydrogen peroxide solution for inactivation of endogenous peroxidase; thirdly; apply citric acid buffer (10 mmol/L pH 6.0) for CXCR4 and CD133 antigen retrieval; fourthly, use $1 \%$ goat serum albumin to seal, and then dropwise add primary antibodies (mouse antihuman CXCR4 monoclonal antibody, British Abcam Corporation; mouse anti-human CD133 monoclonal antibody, American Novus Corporation); fifthly, dropwise add biotin-marked working solution of secondary antibodies (PV-9000 detection kit, Beijing Zhongshan Golden Bridge Biotechnology Co, Ltd.) again after placing $2 \mathrm{~h}$ in room temperature (CD133 detection should be kept staying overnight in a refrigerator at $4^{\circ} \mathrm{C}$; sixthly, incubate in a drying oven for $15 \mathrm{~min}$ at $37^{\circ} \mathrm{C}$, wash with phosphate buffer solution (PBS), and then add dropwise streptavidin working solution marked by horseradish peroxidase (Beijing Zhongshan Golden Bridge Biotechnology Co, Ltd.); seventhly, wash with PBS and use DAB for coloration after incubation for $15 \mathrm{~min}$ at $37^{\circ} \mathrm{C}$, and then flush with flowing water; eighthly, apply hematoxylin for redyeing, $p$-Xylene for transparency and neural resin for sealing. The known positive sections were considered as positive control, and PBS as negative control instead of primary antibodies.

\section{Observation indexes}

CXCR4 and CD133 expression was observed. In follow-up period, routine examination was performed once in 3 months, general CT imageological examination and colonoscopy once in a year. Additionally, the overall survival (OS) of patients was recorded.

\section{Evaluation criteria}

All sections were observed and judged by two pathologists on their own who were unknown to the clinical data of patients. Five high power fields $(\times 400)$ were randomly selected to count the total number of tumor cells and number of positive cells. According to proportion of positive cells, points were scored, namely $0 \%, 1 \% \sim 25 \%, 26 \% \sim 50 \%, 51 \% \sim 75 \%$ and $76 \% \sim 100 \%$ were $0 \sim 4$ points. The positive cells were divided into no, weak and strong expression according to coloration intensity, and 0 3 points were scored. By adding the points of proportion and coloration intensity of positive cells, the section with final points $\geq 4$ was considered as positive expression, otherwise as negative expression.

\section{Statistical data analysis}

SAS 9.3 software package was used for statistical analysis. Fourfold table $\chi^{2}$ test was applied to compare the ratios between two samples, and continuous correction was conducted if the theoretical frequency in some component was less than 5. Line listing $\chi^{2}$ test was used to compare the ratios among many samples, and KaplanMeier survival curve and Log-rank test for survival analysis. Inspection level was $\alpha=0.05$.

\section{Results}

CXCR4 and CD133 expression in tumor tissue and epithelial tissue adjacent to cancer

In epithelial tissue adjacent to cancer, CXCR4 was weakly expressed in cytoplasm or cytomembrane, and the positive expression rate was $8.16 \%$ (4/49); CD133 was only weakly expressed in the local part, and the positive expression rate was $6.12 \%$ (3/49). In the tumor tissue, CXCR4 was expressed in cytoplasm, whereas its expression could also be seen in cytomembrane of some patients. Claybank positive expression rate was $61.22 \%$ (30/49). CD133 expression in the side of lumen close to glands polar was polarly distributed, and the positive expression rate was $36.73 \%$ (18/49) (Figure 1 2).

Relationship between CXCR4, CD133 expression and clinicopathological features of patients with colon cancer

Among 49 patients with phase II III colon cancer, CXCR4 and CD133 expression in tumor tissue was not related to the age, gender, primary focal sites, tumor size, TNM staging, histological types, tumor infiltration depth and presence or absence of lymphatic metastasis, but CXCR4 and CD133 co-expression was associated with TNM staging and lymphatic metastasis (Table 1).

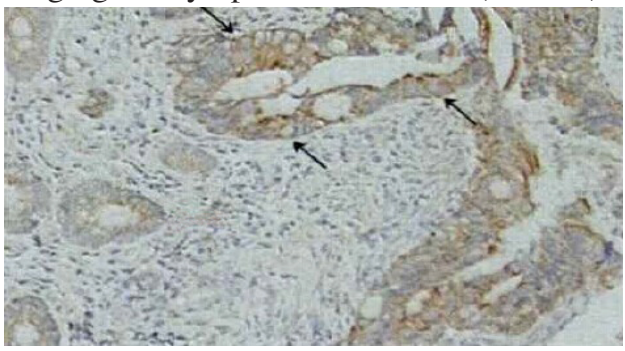

Figure 1. CXCR4 Expression in Tumor Tissue of a Patient with Colon Caner

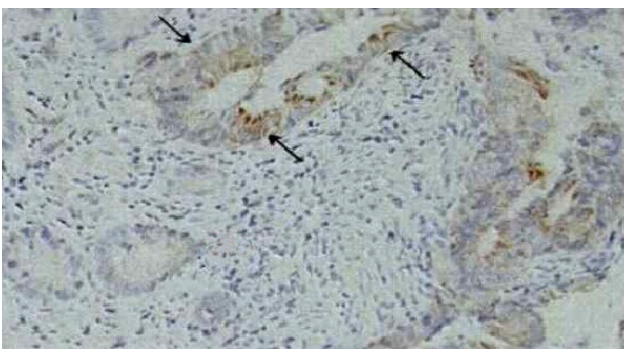

Figure 2. CD133 Expression in Tumor Tissue of a Patient with Colon Caner 
Effect of CXCR4 and CD133 Co-expression on the Prognosis of Patients with Stage II III Colon Cancer Table 1. Relationship between CXCR4, CD133 Expression and Clinicopathological Features of Patients with Colon Cancer

\begin{tabular}{|c|c|c|c|c|c|c|c|c|c|c|c|c|}
\hline \multirow[t]{2}{*}{ Pathological features } & \multicolumn{2}{|c|}{ CXCR4 (n) } & \multirow[t]{2}{*}{$\chi^{2}$} & \multirow[t]{2}{*}{$P$} & \multicolumn{2}{|c|}{ CD133 (n) } & \multirow[t]{2}{*}{$\chi^{2}$} & \multicolumn{3}{|c|}{ P CXCR4 \& CD133(n) } & \multirow{2}{*}{$\chi^{2}$} & \multirow[t]{2}{*}{$P$} \\
\hline & + & - & & & + & - & & Co-e & & Others & & \\
\hline \multicolumn{13}{|l|}{ Age } \\
\hline$<60$ & 13 & 9 & 0.0766 & 0.7820 & 7 & 16 & 0.7402 & 0.3896 & 3 & 19 & 1.7811 & 0.1820 \\
\hline$\geq 60$ & 17 & 10 & & & 11 & 15 & & & 8 & 19 & & \\
\hline \multicolumn{13}{|l|}{ Gender } \\
\hline Male & 19 & 9 & 1.2107 & 0.2712 & 10 & 18 & 0.0293 & 0.8642 & 7 & 21 & 0.2442 & 0.6212 \\
\hline Female & 11 & 10 & & & 8 & 13 & & & 4 & 17 & & \\
\hline \multicolumn{13}{|l|}{ Primary foal sites } \\
\hline Left hemicolon & 17 & 11 & 0.0072 & 0.9325 & 11 & 17 & 0.1829 & 0.6689 & 8 & 20 & 1.4067 & 0.2356 \\
\hline Right hemicolon & 13 & 8 & & & 7 & 14 & & & 3 & 18 & & \\
\hline \multicolumn{13}{|l|}{ Tumor size } \\
\hline$<2$ & 2 & 3 & 1.4213 & 0.4913 & 1 & 4 & 0.8284 & 0.6609 & 0 & 5 & 1.7623 & 0.4143 \\
\hline $2 \sim 5$ & 20 & 10 & & & 11 & 19 & & & 7 & 23 & & \\
\hline$\geq 5$ & 8 & 6 & & & 6 & 8 & & & 4 & 10 & & \\
\hline \multicolumn{13}{|l|}{ TNM staging } \\
\hline Phase II & 13 & 11 & 0.9870 & 0.3205 & 7 & 17 & 1.1593 & 0.2816 & 1 & 23 & 9.0312 & 0.0027 \\
\hline Phase III & 17 & 8 & & & 11 & 14 & & & 10 & 15 & & \\
\hline \multicolumn{13}{|l|}{ Histological types } \\
\hline High differentiation & 3 & 2 & 1.1555 & 0.5612 & 1 & 4 & 0.8796 & 0.6442 & 1 & 4 & 0.1758 & 0.9158 \\
\hline Moderate differentiation & 13 & 11 & & & 10 & 14 & & & 6 & 18 & & \\
\hline Poor differentiation & 14 & 6 & & & 7 & 13 & & & 4 & 16 & & \\
\hline \multicolumn{13}{|l|}{ Lymphatic metastasis } \\
\hline Yes & 19 & 13 & 0.1329 & 0.7154 & 10 & 22 & 1.1939 & 0.2745 & 3 & 29 & 7.0206 & 0.0081 \\
\hline No & 11 & 6 & & & 8 & 9 & & & 8 & 9 & & \\
\hline \multicolumn{13}{|l|}{ Infiltration depth } \\
\hline Serosa and tissue outside ser & 30 & 17 & 1.1525 & 0.2830 & 18 & 29 & 0.1235 & 0.7252 & 11 & 36 & 0.0000 & 1.0000 \\
\hline Not beyond muscular layer & 0 & 2 & & & 0 & 2 & & & 0 & 2 & & \\
\hline
\end{tabular}

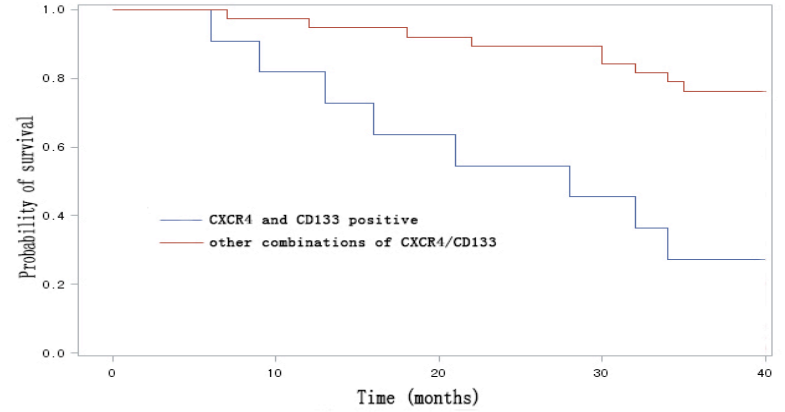

Figure 3. Effect of CXCR4 and CD133 Co-expression on the Survival of Patients with Colon Cancer

Effect of CXCR4 and CD133 expression on the survival of patients with colon cancer

All patients were strictly followed up to evaluate the effect of CXCR4 and CD133 co-expression on the prognosis. The median follow-up time was 26 months. There were 17 deaths in the follow-up period.

The 3-year survival rate of patients with positive expression of CXCR4 was $53.33 \%$ (16/30), while those with negative one being $84.21 \%$ (16/19). There was statistical significance by comparison to them $\left(\chi^{2}=4.8949, p=0.0269\right)$. The 3 -year survival rates of patients respectively with positive and negative expression of CD133 were $50.00 \%$ (9/18) and $74.19 \%$ (23/31), and no significant difference was presented by comparison to them $\left(\chi^{2}=2.9419, p=0.0863\right)$. Besides, the 3 -year survival rate of patients with CXCR4 and CD133 co-expression was $27.27 \%$ (3/11), and that of the rest being $76.32 \%$ $(29 / 38)$. There was statistical significance by comparison to them $\left(\chi^{2}=7.0206, p=0.0081\right)$. Further survival analysis revealed that the survival curve of patients with CXCR4 and CD133 co-expression was significantly lower than that of the rest $(p=0.0004)$, suggesting that CXCR4 and CD133 co-expression is a risk factor that affects the prognosis (Figure 3).

\section{Discussion}

CXCL12-CXCR4 biological axis is coupled molecules closely related to tumor targeted metastasis. By interactions with its specific receptor CXCR4, CXCL12 causes reconstruction of target cytoskeleton and makes target cells migration directly after adhering to endothelial cells firmly. CXCR4 can promote the selective process of metastatic sites, induce cytoskeleton to reconstruct and regulate cancer cell movement. Studies have revealed that CXCR4 expression plays an important role in the invasion and metastasis of various tumors, such as diffused large B cell lymphoma, nasal and facial basal cell carcinoma, triple negative breast cancer and papillary thyroid carcinoma (Wang et al., 2013; Xu et al., 2013; Yu et al., 2013; Moreno et al., 2014). Zhang et al made a meta-analysis on CXCR4 expression and prognosis of breast cancer, and the results showed that CXCR4 was an efficient prognostic factor for breast cancer, and its over-expression was significantly associated with lymph node status and distant metastasis (Zhang et al., 2014). Wu et al analyzed the relationship between CXCR4 and clinicopathological features as well as prognosis of patients with esophageal carcinoma. The results indicated that CXCR4 expression was intimately associated with tumor infiltration depth, TNM staging and histological types, and the clinical outcomes of patients 
with CXCR4 over-expression were poorer (Wu et al., 2014). In this study, the 3-year survival rate of patients with positive expression of CXCR4 was significantly lower than those with negative one, similar to the research results at home and abroad.

CD133, also termed Prominin-1, is a quintic transmembrane glycoprotein with 120-kDa molecular weight. It includes an extracellular $\mathrm{N}$ terminal, 5 transmembrane regions, 4 extracellular loops and $\mathrm{C}$ terminals. CD133, one of the prognostic indicators for colorectal cancer, is a widely-applied tumor stem cell surface marker for colorectal cancer at present (Yao et al., 2014; Zheng et al., 2014). The studies found that the overall survival of patients with high expression of CD133 was markedly lower than those with low expression in the tumors, such as pancreatic cancer and lung cancer (Chen et al., 2014; Zhai et al., 2014; Tan et al., 2014). Wang et al studied the relationship between CD133 expression and prognosis in non-small cell lung cancer (NSCLC), and the results showed that CD133 was only related to tumor differentiated degrees and histological types instead of other clinicopathological features and diseasefree survival (Wang et al., 2014). However, the overall survival of patients with positive expression of CD133 was lower than those with negative one in this study, and the study made by Wu et al also demonstrated that the prognosis of patients with positive expression of CD133 was poorer (Wang et al., 2014), suggesting that CD133 can be considered as a prognostic marker for NSCLC. In this study, no significant difference was presented between the patients with positive expression of CD133 and those with negative one in terms of 3-year survival rate, which might be related to small sample size and shorter follow-up time. Hence, it still needs large-sample studies to further confirm the effect of CD133 on the prognosis of patients with colon cancer. Additionally, this study investigated the effect of CXCR4 and CD133 co-expression on the prognosis of patients with colon cancer, and the results indicated that the survival of patients with CXCR4 and CD133 co-expression went down notably, suggesting that CXCR4 and CD133 co-expression is a risk factor that affects the prognosis.

\section{References}

Bahnassy AA, Fawzy M, El-Wakil M, et al (2014). Aberrant expression of cancer stem cell markers (CD44, CD90, and CD133) contributes to disease progression and reduced survival in hepatoblastoma patients: 4-year survival data. Transl Res, [Epub ahead of print].

Chen S, Song X, Chen Z, et al (2013). CD133 expression and the prognosis of colorectal cancer: a systematic review and meta-analysis. PLoS One, 8, 56380.

Chen K, Li Z, Jiang P, et al (2014). Co-expression of CD133, $\mathrm{CD} 44 \mathrm{v} 6$ and human tissue factor is associated with metastasis and poor prognosis in pancreatic carcinoma. Oncol Rep, 32, 755-63.

Gong JP, Yang L, Huang XE, et al (2014). Outcomes based on risk assessment of anastomotic leakage after rectal cancer surgery. Asian Pac J Cancer Prev, 15, 707-12.

Gupta P, Sharma PK, Mir H, et al (2014). CCR9/CCL25 expression in non-small cell lung cancer correlates with aggressive disease and mediates key steps of metastasis. Oncotarget, 5, 10170-9.
Li J, Jiang K, Qiu X, et al (2014). Overexpression of CXCR4 is significantly associated with cisplatin-based chemotherapy resistance and can be a prognostic factor in epithelial ovarian cancer. BMB Rep, 47, 33-8.

Liu BL, Liu SJ, Baskys A, et al (2014). Platinum sensitivity and CD133 expression as risk and prognostic predictors of central nervous system metastases in patients with epithelial ovarian cancer. BMC Cancer, 14, 829.

Moreno MJ, Bosch R, Dieguez-Gonzalez R, et al (2014). CXCR4 expression enhances diffuse large B-cell lymphoma dissemination and decreases patient survival. J Pathol, [Epub ahead of print].

Nakata B, Fukunaga S, Noda E, et al (2008). Chemokine receptor CCR7 expression correlates with lymph node metastasis in pancreatic cancer. Oncology, 74, 69-75.

Oliver JA, Ortiz R, Melguizo C, et al (2014). Prognostic impact of MGMT promoter methylation and MGMT and CD133 expression in colorectal adenocarcinoma. BMC Cancer, $\mathbf{1 4}$, 511.

Tan Y, Chen B, Xu W, et al (2014). Clinicopathological significance of CD133 in lung cancer: A meta-analysis. Mol Clin Oncol, 2, 111-5.

Wang N, Luo HJ, Yin GB, et al (2013). Overexpression of HIF$2 \alpha$, TWIST, and CXCR4 is associated with lymph node metastasis in papillary thyroid carcinoma. Clin Dev Immunol, 2013, 589423.

Wu J, Wu X, Liang W, et al (2014). Clinicopathological and prognostic significance of chemokine receptor CXCR4 overexpression in patients with esophageal cancer: a metaanalysis. Tumour Biol, 35, 3709-15.

Wang W, Chen Y, Deng J, et al (2014). The prognostic value of CD133 expression in non-small cell lung cancer: a metaanalysis. Tumour Biol, 35, 9769-75.

Wu H, Qi XW, Yan GN, et al (2014). Is CD133 expression a prognostic biomarker of non-small-cell lung cancer? A systematic review and meta-analysis. PLoS One, 9, 100168.

Wu XY, Huang XE, Cao J, et al (2014). A predictive model for evaluating responsiveness to pemetrexed treatment in patients with advanced colorectal cancer. Asian Pac J Cancer Prev, 15, 5941-4.

Wu XY, Huang XE, You SX, et al (2013). Phase II study of pemetrexed as second or third line combined chemotherapy in patients with colorectal cancer. Asian Pac J Cancer Prev, 14, 2019-22.

Xu CZ, Wang PH, Yan XJ, et al (2013). Expression of CXCR4 is associated with progression and invasion in patients with nasal-surface basal cell carcinoma.J Otorhinolaryngol Relat Spec, 75, 332-41.

Yao Q, Sun JG, Ma H, et al (2014). Monitoring microRNAs using a molecular beacon in CD133+/ CD338+ human lung adenocarcinoma-initiating A549 cells. Asian Pac J Cancer Prev, 15, 161-6.

Yang L, Huang XE, Xu L, et al (2013). Role of MYH polymorphisms in sporadic colorectal cancer in China: a case-control, population-based study. Asian Pac J Cancer Prev, 14, 6403-9.

Yu S, Wang X, Liu G, et al (2013). High level of CXCR4 in triple-negative breast cancer specimens associated with a poor clinical outcome. Acta Med Okayama, 67, 369-75.

Zhang Z, Ni C, Chen W, et al (2014). Expression of CXCR4 and breast cancer prognosis: a systematic review and metaanalysis. BMC Cancer, 14, 49.

Zhai JH, Gu WC, Xu XL, et al (2014). Prognostic value of CD133 expression in cancer patients treated with chemoradiotherapy: a meta-analysis. Tumour Biol, [Epub ahead of print].

Zheng NG, Mo SJ, Li JP, et al (2014). Anti-CSC effects in human esophageal squamous cell carcinomas and Eca109/9706 cells induced by nanoliposomal quercetin alone or combined with CD 133 antiserum. Asian Pac J Cancer Prev, 15, 8679-84. 